\title{
Simulation and Modeling of Micro-Fibrous Web Formation in Melt Blowing
}

Guangwu Sun ${ }^{1,3}$, Jingru Yang ${ }^{2}$, XiaoXia Sun ${ }^{1,3}$, Xinhou Wang ${ }^{1,3^{*}}$

1 College of Textiles, Donghua University, Shanghai, P.R.

China

2 College of Textiles, Zhongyuan University of Technology,

Zhengzhou, P.R. China

3 Key Laboratory of Textile Science \& Technology of

Eco-Textile, Ministry of Education, Donghua University,

Shanghai, P.R. China

\section{Corresponding Author:}

Xinhou Wang, College of Textiles, Donghua University,

Shanghai, P.R. China.

E-mail address: xhwang@dhu.edu.cn 


\section{Supporting information}

S1. Calculated MB air flow velocity field
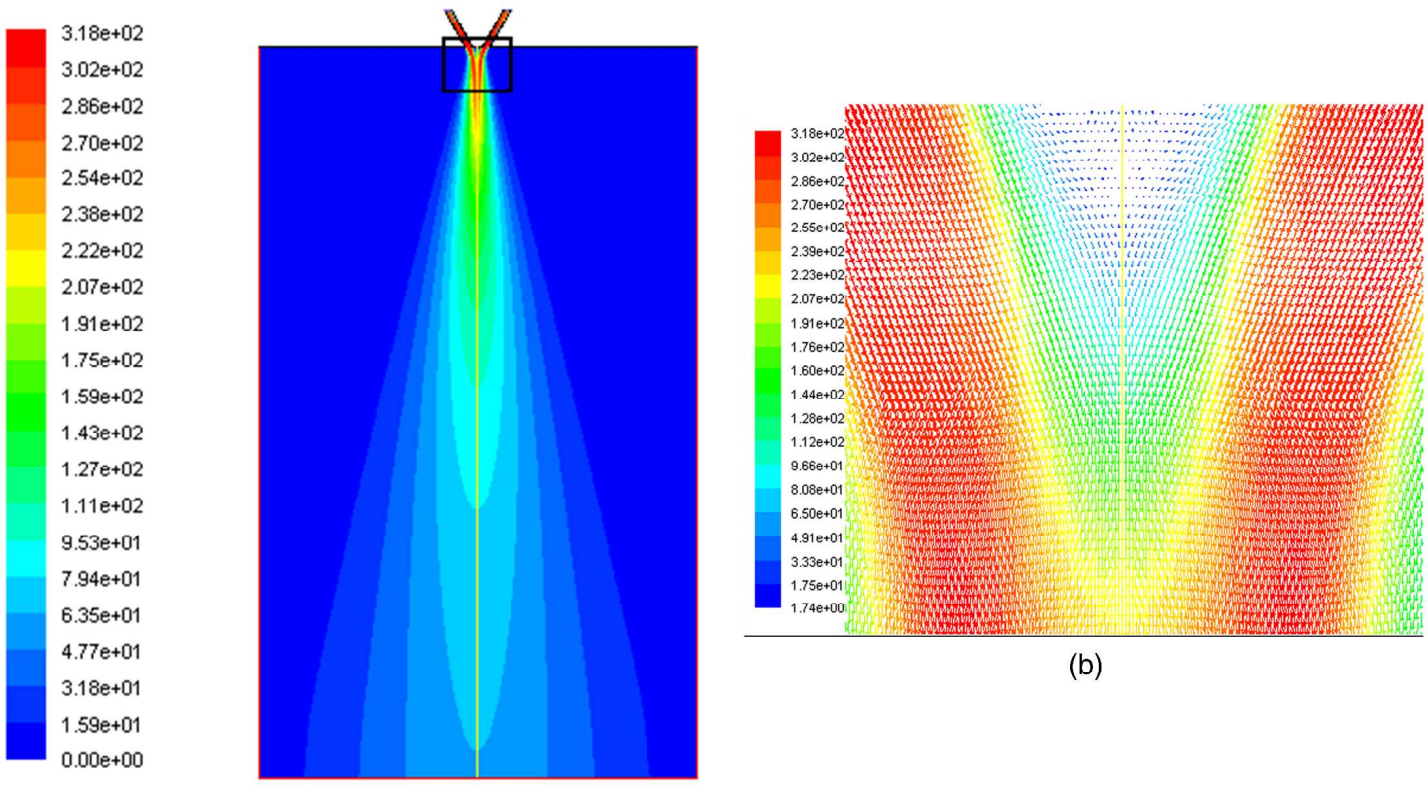

(b)

(a)

Figure S1. Calculated MB air flow velocity field

S2. Distribution parameters obtained from image analysis data

Table S1. Distribution parameters

\begin{tabular}{|c|c|c|c|}
\hline DCD $(\mathrm{cm})$ & $\mathrm{Sx} 2$ & $\mathrm{Sy} 2$ & $\begin{array}{c}\text { association } \\
\text { coefficient }\end{array}$ \\
\hline 4 & 4545.092471 & 5218.082766 & -0.007949545 \\
\hline 5 & 6808.812414 & 7172.598048 & -0.005361057 \\
\hline 10 & 18395.21006 & 18319.66228 & 0.005559283 \\
\hline 15 & 44505.18216 & 44162.93237 & 0.011858895 \\
\hline 20 & 64067.08001 & 65130.12451 & 0.019983915 \\
\hline 25 & 81102.25805 & 80679.00555 & 0.030063625 \\
\hline
\end{tabular}

S3. Experimental and predicted basis weight for single orifice Experiment data (basis weight) (g)

Table S2. Sample 1

\begin{tabular}{|l|l|l|l|l|l|}
\hline 0.027 & 0.025 & 0.021 & 0.023 & 0.021 & 0.03 \\
\hline
\end{tabular}




\begin{tabular}{|l|l|l|l|l|l|}
\hline 0.099 & 0.097 & 0.093 & 0.107 & 0.122 & 0.104 \\
\hline 0.102 & 0.103 & 0.085 & 0.08 & 0.111 & 0.09 \\
\hline 0.011 & 0.013 & 0.015 & 0.014 & 0.02 & 0.017 \\
\hline
\end{tabular}

Table S3. Sample 2

\begin{tabular}{|l|l|l|l|l|l|}
\hline 0.024 & 0.023 & 0.028 & 0.032 & 0.019 & 0.023 \\
\hline 0.109 & 0.097 & 0.107 & 0.14 & 0.098 & 0.103 \\
\hline 0.077 & 0.087 & 0.088 & 0.108 & 0.101 & 0.085 \\
\hline 0.018 & 0.013 & 0.018 & 0.014 & 0.02 & 0.011 \\
\hline
\end{tabular}

Table S4.Sample 3

\begin{tabular}{|l|l|l|l|l|l|}
\hline 0.03 & 0.033 & 0.026 & 0.024 & 0.017 & 0.024 \\
\hline 0.114 & 0.107 & 0.111 & 0.097 & 0.09 & 0.103 \\
\hline 0.089 & 0.087 & 0.099 & 0.07 & 0.08 & 0.083 \\
\hline 0.01 & 0.014 & 0.014 & 0.011 & 0.021 & 0.015 \\
\hline
\end{tabular}

Table S5.Sample 4

\begin{tabular}{|l|l|l|l|l|l|}
\hline 0.027 & 0.027 & 0.017 & 0.033 & 0.023 & 0.015 \\
\hline 0.122 & 0.091 & 0.117 & 0.12 & 0.082 & 0.098 \\
\hline 0.072 & 0.083 & 0.088 & 0.086 & 0.112 & 0.09 \\
\hline 0.013 & 0.02 & 0.021 & 0.017 & 0.019 & 0.021 \\
\hline
\end{tabular}

Table S6. Predicted data (beads number)

\begin{tabular}{|l|l|l|l|l|l|}
\hline 5 & 3 & 2 & 4 & 7 & 9 \\
\hline 25 & 18 & 26 & 18 & 26 & 21 \\
\hline 23 & 25 & 21 & 26 & 25 & 24 \\
\hline 4 & 8 & 9 & 6 & 3 & 4 \\
\hline
\end{tabular}

S4. Experimental and predicted basis weight for multiple orifice Experiment data (basis weight) $(\mathrm{g})$

Table S7. Sample 5

\begin{tabular}{|l|l|l|l|l|l|}
\hline 0.012 & 0.012 & 0.011 & 0.011 & 0.009 & 0.009 \\
\hline 0.102 & 0.087 & 0.068 & 0.061 & 0.080 & 0.076 \\
\hline 0.133 & 0.096 & 0.087 & 0.086 & 0.093 & 0.106 \\
\hline 0.130 & 0.103 & 0.052 & 0.105 & 0.081 & 0.071 \\
\hline 0.019 & 0.013 & 0.011 & 0.020 & 0.012 & 0.003 \\
\hline
\end{tabular}

Table S8. Sample 6 


\begin{tabular}{|l|l|l|l|l|l|}
\hline 0.008 & 0.009 & 0.011 & 0.010 & 0.011 & 0.012 \\
\hline 0.065 & 0.066 & 0.079 & 0.071 & 0.087 & 0.082 \\
\hline 0.070 & 0.120 & 0.136 & 0.103 & 0.095 & 0.161 \\
\hline 0.090 & 0.119 & 0.128 & 0.116 & 0.076 & 0.112 \\
\hline 0.028 & 0.018 & 0.027 & 0.034 & 0.015 & 0.042 \\
\hline
\end{tabular}

Table S9. Predicted data (beads number)

\begin{tabular}{|l|l|l|l|l|l|}
\hline 103 & 103 & 100 & 100 & 101 & 103 \\
\hline 475 & 505 & 486 & 508 & 526 & 497 \\
\hline 736 & 813 & 864 & 803 & 824 & 667 \\
\hline 493 & 524 & 494 & 489 & 500 & 487 \\
\hline 100 & 101 & 104 & 105 & 102 & 104 \\
\hline
\end{tabular}

S5. Predicted fibrous web collected with stationary collector

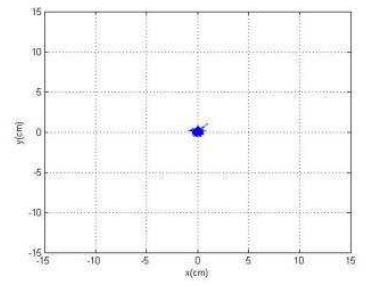

(a) $\mathrm{DCD}=1 \mathrm{~cm}$;

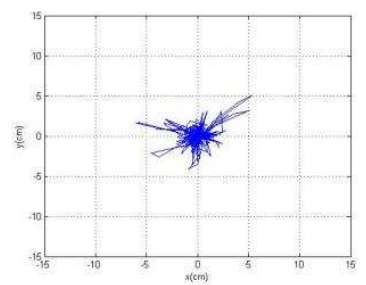

(e) $\mathrm{DCD}=10 \mathrm{~cm}$;

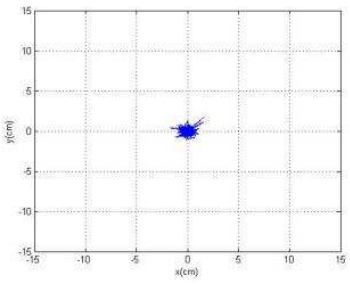

(b) $\mathrm{DCD}=2 \mathrm{~cm}$;

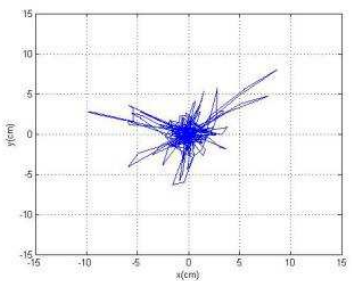

(f) $\mathrm{DCD}=20 \mathrm{~cm}$;

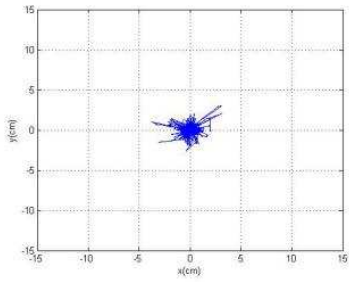

(c) $\mathrm{DCD}=5 \mathrm{~cm}$

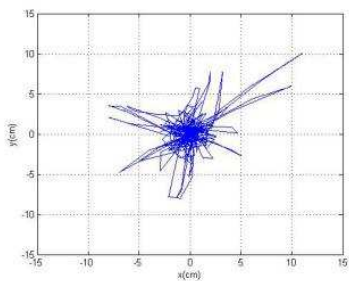

(g) $\mathrm{DCD}=30 \mathrm{~cm}$

Figure S2. Predicted fibrous web collected under different DCD with stationary collector

S6. predicted initial whippings amplitude

Table S10. Lateral displacements (400 beads)

\begin{tabular}{|l|l|l|}
\hline Beads Number & X displacement $(\mathrm{mm})$ & Y displacement $(\mathrm{mm})$ \\
\hline 1 & 0.06302352 & 0.022756146 \\
\hline 2 & 0.214961786 & 0.026891065 \\
\hline 3 & -0.264774373 & -0.1373673 \\
\hline 4 & 0.101061035 & -0.136714506 \\
\hline
\end{tabular}




\begin{tabular}{|c|c|c|}
\hline 5 & 0.037364581 & -0.058866562 \\
\hline 6 & -0.15328279 & 0.035924797 \\
\hline 7 & -0.050824187 & 0.142159169 \\
\hline 8 & 0.040161279 & -0.040560048 \\
\hline 9 & 0.419447561 & 0.087891651 \\
\hline 10 & 0.324624022 & 0.008294717 \\
\hline 11 & -0.158229172 & 0.16358086 \\
\hline 12 & 0.355743443 & -0.037894355 \\
\hline 13 & 0.085029425 & -0.090989568 \\
\hline 14 & -0.007391079 & -0.1755301 \\
\hline 15 & 0.083779741 & 0.006050403 \\
\hline 16 & -0.024025427 & 0.101179932 \\
\hline 17 & -0.01455178 & -0.032716973 \\
\hline 18 & 0.174617305 & -0.090509863 \\
\hline 19 & 0.165162247 & -0.061421065 \\
\hline 20 & 0.166118492 & -0.155513555 \\
\hline 21 & 0.078710618 & -0.070516504 \\
\hline 22 & -0.141537525 & -0.000451133 \\
\hline 23 & 0.084072284 & -0.007066871 \\
\hline 24 & 0.191090655 & -0.036090936 \\
\hline 25 & 0.057306471 & 0.064348753 \\
\hline 26 & 0.121283208 & -0.005045387 \\
\hline 27 & 0.085203012 & 0.20358958 \\
\hline 28 & -0.035568317 & 0.042505753 \\
\hline 29 & 0.034446617 & 0.210020533 \\
\hline 30 & -0.092282622 & -0.072463491 \\
\hline 31 & 0.104134725 & 0.047258508 \\
\hline 32 & -0.13445567 & -0.010987233 \\
\hline 33 & -0.125289372 & 0.090233765 \\
\hline 34 & -0.094886693 & 0.084960997 \\
\hline 35 & -0.345119011 & -0.209199386 \\
\hline 36 & 0.168602063 & -0.183361043 \\
\hline 37 & 0.038117733 & -0.177498808 \\
\hline 38 & -0.088490139 & 0.061104408 \\
\hline 39 & 0.160621751 & 0.151352864 \\
\hline 40 & -0.200618154 & -0.009060955 \\
\hline 41 & -0.011984513 & 0.098642367 \\
\hline 42 & -0.028301604 & 0.069265387 \\
\hline 43 & 0.037416332 & -0.13096709 \\
\hline 44 & 0.036672224 & 0.041924518 \\
\hline
\end{tabular}




\begin{tabular}{|c|c|c|}
\hline 45 & -0.101378293 & -0.074538327 \\
\hline 46 & -0.003522511 & 0.181314389 \\
\hline 47 & -0.01932656 & -0.001001999 \\
\hline 48 & 0.073577721 & 0.184097177 \\
\hline 49 & 0.128148896 & -0.069784214 \\
\hline 50 & 0.130025256 & 0.050897143 \\
\hline 51 & -0.101234457 & 0.007604219 \\
\hline 52 & 0.00906777 & -0.242436278 \\
\hline 53 & -0.142314685 & -0.0958878 \\
\hline 54 & -0.130520783 & 0.092488381 \\
\hline 55 & -0.000802855 & -0.006424565 \\
\hline 56 & 0.17964973 & -0.160170323 \\
\hline 57 & -0.09021763 & -0.061236353 \\
\hline 58 & 0.043531766 & 0.02326151 \\
\hline 59 & -0.026442239 & -0.114459048 \\
\hline 60 & 0.1309727 & 0.0967223 \\
\hline 61 & -0.127656426 & -0.057364081 \\
\hline 62 & 0.003816276 & 0.215107508 \\
\hline 63 & 0.064765345 & -0.138410428 \\
\hline 64 & 0.1290098 & 0.004518503 \\
\hline 65 & 0.181007285 & -0.208339544 \\
\hline 66 & 0.010072556 & -0.087782339 \\
\hline 67 & -0.174839161 & -0.044671844 \\
\hline 68 & -0.087010106 & 0.06082319 \\
\hline 69 & -0.124435013 & 0.13088305 \\
\hline 70 & 0.27551263 & -0.005242761 \\
\hline 71 & -0.072158766 & -0.033725689 \\
\hline 72 & 0.087687025 & 0.084161769 \\
\hline 73 & -0.022554646 & 0.098616037 \\
\hline 74 & 0.104159902 & -0.001194655 \\
\hline 75 & -0.089653035 & 0.081476231 \\
\hline 76 & -0.164369216 & 0.073721481 \\
\hline 77 & -0.166726085 & -0.092824733 \\
\hline 78 & 0.057224436 & 0.082035286 \\
\hline 79 & -0.020791315 & -0.075260825 \\
\hline 80 & -0.022980725 & -0.068581371 \\
\hline 81 & 0.166366726 & -0.00370235 \\
\hline 82 & 0.034178532 & -0.041759601 \\
\hline 83 & 0.023186741 & -0.019173803 \\
\hline 84 & 0.186104706 & 0.043243757 \\
\hline
\end{tabular}




\begin{tabular}{|c|c|c|}
\hline 85 & -0.094296773 & 0.138873992 \\
\hline 86 & 0.081655953 & -0.035775681 \\
\hline 87 & 0.097886204 & 0.024478211 \\
\hline 88 & -0.028567463 & -0.024154891 \\
\hline 89 & 0.025280117 & 0.023607057 \\
\hline 90 & -0.136656274 & 0.072833682 \\
\hline 91 & -0.134559134 & -0.05349215 \\
\hline 92 & 0.012293059 & 0.030937132 \\
\hline 93 & 0.08466017 & 0.059051006 \\
\hline 94 & 0.303062522 & -0.034171344 \\
\hline 95 & -0.078170664 & 0.217907346 \\
\hline 96 & 0.021958308 & -0.075833549 \\
\hline 97 & -0.009669717 & -0.086440499 \\
\hline 98 & -0.226582395 & -0.174867009 \\
\hline 99 & -0.051454125 & 0.116229975 \\
\hline 100 & -0.210366171 & 0.134744263 \\
\hline 101 & 0.098505971 & -0.024210155 \\
\hline 102 & -0.104092111 & 0.122645133 \\
\hline 103 & 0.011732543 & 0.02015457 \\
\hline 104 & -0.063827836 & 0.044186637 \\
\hline 105 & 0.035577679 & 0.008163193 \\
\hline 106 & -0.070368245 & 0.062946678 \\
\hline 107 & 0.057432074 & 0.003560138 \\
\hline 108 & 0.08666564 & 0.319423536 \\
\hline 109 & 0.200661684 & -0.16901778 \\
\hline 110 & -0.022754503 & -0.21770192 \\
\hline 111 & -0.250650757 & -0.09864252 \\
\hline 112 & -0.098413747 & -0.11569226 \\
\hline 113 & 0.158780956 & -0.075370073 \\
\hline 114 & -0.125674409 & -0.001352918 \\
\hline 115 & 0.112639756 & -0.042847648 \\
\hline 116 & 0.014540697 & 0.062377433 \\
\hline 117 & 0.168404709 & 0.021309358 \\
\hline 118 & -0.229850052 & 0.123835137 \\
\hline 119 & -0.023173516 & 0.215481539 \\
\hline 120 & -0.141579554 & 0.166934865 \\
\hline 121 & 0.340866846 & -0.203819677 \\
\hline 122 & 0.096729362 & -0.292108039 \\
\hline 123 & 0.161638422 & 0.083444706 \\
\hline 124 & -0.124036303 & -0.20037891 \\
\hline
\end{tabular}




\begin{tabular}{|c|c|c|}
\hline 125 & -0.05492953 & 0.016147979 \\
\hline 126 & -0.031937941 & 0.008991318 \\
\hline 127 & 0.128753611 & 0.246358987 \\
\hline 128 & -0.032571206 & -0.003397246 \\
\hline 129 & 0.082232312 & -0.072748096 \\
\hline 130 & -0.240506953 & 0.063968689 \\
\hline 131 & -0.041477097 & 0.035491077 \\
\hline 132 & -0.096538021 & 0.022742629 \\
\hline 133 & -0.184857279 & -0.045006311 \\
\hline 134 & 0.059543067 & -0.154649749 \\
\hline 135 & 0.033053216 & 0.032810096 \\
\hline 136 & 0.003924398 & -0.160699548 \\
\hline 137 & -0.156329208 & -0.099766611 \\
\hline 138 & 0.132160824 & 0.139023866 \\
\hline 139 & 0.041046844 & -0.056066001 \\
\hline 140 & -0.035055506 & -0.01150528 \\
\hline 141 & 0.002683064 & 0.106888692 \\
\hline 142 & -0.030710217 & -0.031205212 \\
\hline 143 & -0.205153962 & 0.153316812 \\
\hline 144 & -0.033483039 & -0.03615199 \\
\hline 145 & -0.097449965 & 0.135286424 \\
\hline 146 & -0.114779244 & 0.092147684 \\
\hline 147 & -0.135549483 & -0.005192107 \\
\hline 148 & -0.062541756 & -0.093896727 \\
\hline 149 & -0.234742174 & -0.089380093 \\
\hline 150 & 0.113023705 & -0.049049594 \\
\hline 151 & 0.060959683 & -0.061328233 \\
\hline 152 & -0.002347597 & -0.048377572 \\
\hline 153 & -0.004075749 & 0.117878775 \\
\hline 154 & -0.09355803 & -0.02154868 \\
\hline 155 & 0.119406836 & 0.118564476 \\
\hline 156 & -0.015615301 & -0.061058973 \\
\hline 157 & -0.083754804 & 0.128447405 \\
\hline 158 & 0.15840486 & -0.085882977 \\
\hline 159 & -0.026346901 & 0.024981459 \\
\hline 160 & -0.069043987 & 0.126036518 \\
\hline 161 & -0.034432801 & -0.044226325 \\
\hline 162 & -0.09939104 & -0.037440161 \\
\hline 163 & -0.131297643 & 0.258443118 \\
\hline 164 & 0.296089123 & 0.069251968 \\
\hline
\end{tabular}




\begin{tabular}{|c|c|c|}
\hline 165 & 0.194051817 & -0.080436475 \\
\hline 166 & 0.036048229 & 0.072001545 \\
\hline 167 & -0.147355153 & -0.02096681 \\
\hline 168 & -0.101447229 & 0.099287632 \\
\hline 169 & -0.020692731 & 0.171900818 \\
\hline 170 & 0.092767108 & -0.205140255 \\
\hline 171 & -0.156133044 & 0.145948143 \\
\hline 172 & -0.273099132 & 0.214548972 \\
\hline 173 & -0.169858273 & 0.030980919 \\
\hline 174 & 0.03909301 & 0.202384107 \\
\hline 175 & 0.045873143 & 0.011689037 \\
\hline 176 & 0.052944331 & -0.155398093 \\
\hline 177 & -0.015271525 & -0.259260721 \\
\hline 178 & 0.021531413 & -0.042962864 \\
\hline 179 & -0.055813043 & 0.093398067 \\
\hline 180 & 0.101043252 & 0.022783014 \\
\hline 181 & -0.159613211 & 0.073109604 \\
\hline 182 & 0.053337022 & -0.076758683 \\
\hline 183 & -0.099482837 & 0.03199969 \\
\hline 184 & -0.039254312 & -0.189531649 \\
\hline 185 & 0.06479539 & -0.100286975 \\
\hline 186 & 0.121798685 & -0.115783808 \\
\hline 187 & -0.131005819 & 0.081497946 \\
\hline 188 & 0.147770141 & -0.023716435 \\
\hline 189 & 0.077379742 & -0.149312408 \\
\hline 190 & -0.00795497 & $5.02 \mathrm{E}-05$ \\
\hline 191 & -0.022883167 & -0.078837095 \\
\hline 192 & -0.025507079 & -0.075690306 \\
\hline 193 & -0.035529248 & 0.108331606 \\
\hline 194 & 0.00270133 & 0.01312067 \\
\hline 195 & 0.006012082 & 0.046601839 \\
\hline 196 & 0.096828281 & 0.090964334 \\
\hline 197 & 0.178987032 & -0.005188893 \\
\hline 198 & 0.054730128 & 0.057521506 \\
\hline 199 & -0.024581887 & 0.085096407 \\
\hline 200 & 0.073282695 & 0.128651119 \\
\hline 201 & 0.021477279 & 0.053536743 \\
\hline 202 & -0.120705861 & 0.186378577 \\
\hline 203 & 0.111264566 & -0.013890521 \\
\hline 204 & 0.035992757 & -0.011073059 \\
\hline
\end{tabular}




\begin{tabular}{|c|c|c|}
\hline 205 & 0.015844749 & 0.200493525 \\
\hline 206 & 0.060395429 & -0.06977768 \\
\hline 207 & 0.030641163 & -0.004908082 \\
\hline 208 & -0.110357771 & 0.126129682 \\
\hline 209 & -0.019028672 & 0.020698771 \\
\hline 210 & -0.017120029 & 0.170066731 \\
\hline 211 & -0.06236057 & 0.132597662 \\
\hline 212 & 0.197170482 & 0.005372653 \\
\hline 213 & -0.102650026 & -0.077425418 \\
\hline 214 & -0.05671116 & 0.076022894 \\
\hline 215 & -0.08345876 & -0.152413093 \\
\hline 216 & -0.13763719 & 0.049667055 \\
\hline 217 & -0.022533665 & 0.099751802 \\
\hline 218 & -0.032125583 & 0.030190593 \\
\hline 219 & 0.179349914 & 0.078151004 \\
\hline 220 & -0.029189836 & 0.247452978 \\
\hline 221 & -0.124743489 & 0.128758887 \\
\hline 222 & 0.18795183 & 0.003807441 \\
\hline 223 & 0.144724905 & 0.054933194 \\
\hline 224 & -0.026916034 & 0.054744334 \\
\hline 225 & -0.176546936 & -0.130440792 \\
\hline 226 & -0.052117766 & -0.041880832 \\
\hline 227 & -0.01827888 & 0.148748227 \\
\hline 228 & 0.032359785 & -0.010020554 \\
\hline 229 & -0.030612717 & 0.074007602 \\
\hline 230 & 0.051976414 & -0.127756302 \\
\hline 231 & 0.045936511 & 0.000844782 \\
\hline 232 & -0.146600343 & 0.093428409 \\
\hline 233 & -0.111116766 & -0.145773556 \\
\hline 234 & -0.086869945 & 0.054394264 \\
\hline 235 & -0.059524652 & -0.012808404 \\
\hline 236 & -0.037576774 & -0.106422128 \\
\hline 237 & 0.001461579 & -0.004693078 \\
\hline 238 & -0.355069901 & -0.173172121 \\
\hline 239 & -0.053569707 & -0.245739754 \\
\hline 240 & 0.145635591 & -0.162038173 \\
\hline 241 & -0.125035123 & -0.099465305 \\
\hline 242 & 0.109448451 & -0.156179821 \\
\hline 243 & 0.04106344 & -0.211849526 \\
\hline 244 & -0.003399957 & 0.034873176 \\
\hline
\end{tabular}




\begin{tabular}{|c|c|c|}
\hline 245 & 0.021386425 & -0.19326763 \\
\hline 246 & -0.183450562 & 0.040488706 \\
\hline 247 & -0.009909431 & 0.095321643 \\
\hline 248 & 0.188009155 & -0.028292021 \\
\hline 249 & 0.011527993 & 0.009383174 \\
\hline 250 & 0.004849675 & -0.0458118 \\
\hline 251 & -0.086056815 & -0.163955668 \\
\hline 252 & -0.003611881 & -0.004686205 \\
\hline 253 & 0.027234929 & 0.110502313 \\
\hline 254 & 0.049979704 & 0.199805958 \\
\hline 255 & -0.043699377 & -0.044780585 \\
\hline 256 & -0.027716405 & -0.189899144 \\
\hline 257 & 0.237210187 & -0.015526772 \\
\hline 258 & -0.264716598 & 0.08432466 \\
\hline 259 & 0.261328067 & -0.06601618 \\
\hline 260 & 0.039568073 & -0.142886849 \\
\hline 261 & 0.11722374 & 0.22389303 \\
\hline 262 & -0.19506772 & -0.252254702 \\
\hline 263 & -0.069161852 & -0.050495623 \\
\hline 264 & -0.032593739 & -0.152722844 \\
\hline 265 & 0.049549301 & -0.083233891 \\
\hline 266 & -0.195775265 & 0.067082635 \\
\hline 267 & 0.055283377 & 0.008218806 \\
\hline 268 & -0.142165838 & -0.063550533 \\
\hline 269 & 0.007758573 & 0.091488013 \\
\hline 270 & 0.076466946 & 0.062811069 \\
\hline 271 & 0.038336861 & 0.071474393 \\
\hline 272 & 0.12690263 & -0.069666777 \\
\hline 273 & 0.117928949 & 0.107445932 \\
\hline 274 & -0.076297199 & 0.09015181 \\
\hline 275 & 0.030131252 & 0.294637197 \\
\hline 276 & -0.110696766 & 0.14330121 \\
\hline 277 & -0.154935571 & 0.161039657 \\
\hline 278 & 0.108404962 & -0.009843089 \\
\hline 279 & $5.84 \mathrm{E}-06$ & -0.153619201 \\
\hline 280 & -0.006437409 & -0.043302257 \\
\hline 281 & 0.10679925 & -0.10627448 \\
\hline 282 & 0.069695086 & -0.077633237 \\
\hline 283 & 0.041049395 & 0.060071692 \\
\hline 284 & 0.146550212 & -0.088232638 \\
\hline
\end{tabular}




\begin{tabular}{|c|c|c|}
\hline 285 & 0.108986769 & -0.122973816 \\
\hline 286 & 0.028104236 & -0.052994933 \\
\hline 287 & -0.080921789 & -0.007120031 \\
\hline 288 & -0.07637291 & 0.060191141 \\
\hline 289 & 0.139734141 & -0.134415957 \\
\hline 290 & -0.188933295 & 0.065999039 \\
\hline 291 & -0.002867345 & 0.009730016 \\
\hline 292 & -0.228437261 & 0.191963787 \\
\hline 293 & 0.119619319 & -0.043248635 \\
\hline 294 & 0.101007464 & -0.03109149 \\
\hline 295 & 0.000136215 & -0.139684757 \\
\hline 296 & -0.008303298 & -0.16393147 \\
\hline 297 & -0.291433775 & 0.080466903 \\
\hline 298 & 0.06812305 & -0.039902468 \\
\hline 299 & -0.256989791 & 0.098372447 \\
\hline 300 & -0.271858177 & -0.065908954 \\
\hline 301 & 0.009369559 & 0.22616123 \\
\hline 302 & -0.111177726 & 0.031147209 \\
\hline 303 & 0.048233536 & 0.117650911 \\
\hline 304 & 0.079353044 & -0.038885565 \\
\hline 305 & 0.100540502 & -0.034363186 \\
\hline 306 & -0.08101533 & 0.094354017 \\
\hline 307 & 0.052674522 & 0.058412446 \\
\hline 308 & 0.0117959 & -0.135329954 \\
\hline 309 & 0.096829126 & -0.197182138 \\
\hline 310 & 0.062846516 & -0.140270766 \\
\hline 311 & 0.105247438 & -0.184496352 \\
\hline 312 & -0.01546531 & 0.009408202 \\
\hline 313 & -0.017254456 & -0.046462625 \\
\hline 314 & 0.118127783 & -0.061488383 \\
\hline 315 & -0.248927696 & -0.125164293 \\
\hline 316 & -0.059145908 & 0.101767162 \\
\hline 317 & -0.148934775 & 0.074594072 \\
\hline 318 & -0.044845294 & -0.004000886 \\
\hline 319 & 0.076035985 & 0.110423849 \\
\hline 320 & 0.096788938 & -0.118635652 \\
\hline 321 & -0.118968254 & 0.067180616 \\
\hline 322 & -0.055217219 & 0.049776972 \\
\hline 323 & 0.016061591 & 0.039248406 \\
\hline 324 & -0.034211236 & -0.081850587 \\
\hline
\end{tabular}




\begin{tabular}{|c|c|c|}
\hline 325 & 0.035378148 & 0.033696376 \\
\hline 326 & 0.04687855 & -0.068379145 \\
\hline 327 & -0.109006942 & -0.090636446 \\
\hline 328 & -0.020727444 & -0.140379625 \\
\hline 329 & -0.249916903 & 0.160997133 \\
\hline 330 & 0.134255418 & -0.120878046 \\
\hline 331 & -0.073739887 & -0.009624663 \\
\hline 332 & -0.141111214 & -0.123075959 \\
\hline 333 & -0.029766535 & -0.030990071 \\
\hline 334 & -0.167461144 & -0.360402645 \\
\hline 335 & -0.002444859 & -0.129237125 \\
\hline 336 & -0.065719251 & -0.160281721 \\
\hline 337 & 0.255271839 & -0.159009525 \\
\hline 338 & 0.133447054 & -0.045321465 \\
\hline 339 & -0.292676573 & 0.004837037 \\
\hline 340 & 0.051730847 & 0.224125172 \\
\hline 341 & -0.163884983 & -0.043744001 \\
\hline 342 & -0.029896704 & -0.025355438 \\
\hline 343 & 0.019270888 & -0.189987147 \\
\hline 344 & 0.087646849 & -0.069985353 \\
\hline 345 & -0.032005638 & -0.154760101 \\
\hline 346 & 0.184768561 & -0.02393079 \\
\hline 347 & -0.056373823 & 0.110119871 \\
\hline 348 & 0.038389861 & 0.042477642 \\
\hline 349 & 0.077917881 & -0.095152374 \\
\hline 350 & 0.009985518 & -0.195903317 \\
\hline 351 & 0.1032623 & 0.158688676 \\
\hline 352 & 0.037885949 & 0.23878541 \\
\hline 353 & -0.091914958 & 0.028069424 \\
\hline 354 & -0.211619745 & -0.08648295 \\
\hline 355 & 0.217857966 & 0.110337999 \\
\hline 356 & -0.070860968 & -0.060101031 \\
\hline 357 & 0.012115476 & -0.057822907 \\
\hline 358 & 0.066012522 & 0.09584509 \\
\hline 359 & 0.013315455 & -0.167149063 \\
\hline 360 & -0.10604894 & -0.217501241 \\
\hline 361 & -0.054823918 & 0.058332044 \\
\hline 362 & -0.014639176 & 0.049963518 \\
\hline 363 & 0.173358502 & -0.014498442 \\
\hline 364 & -0.100901898 & 0.074262688 \\
\hline
\end{tabular}




\begin{tabular}{|c|c|c|}
\hline 365 & 0.091976178 & 0.115328079 \\
\hline 366 & 0.036175758 & 0.110316835 \\
\hline 367 & -0.027412282 & -0.063705997 \\
\hline 368 & -0.123894763 & 0.115044512 \\
\hline 369 & -0.03330604 & 0.02562176 \\
\hline 370 & -0.010161541 & -0.058762656 \\
\hline 371 & -0.172237511 & -0.11660618 \\
\hline 372 & 0.022526951 & 0.073782296 \\
\hline 373 & -0.096386431 & -0.027795219 \\
\hline 374 & -0.011046562 & 0.007183246 \\
\hline 375 & 0.039409788 & -0.100420448 \\
\hline 376 & -0.106040483 & -0.169065286 \\
\hline 377 & -0.033788434 & 0.025495623 \\
\hline 378 & 0.04103317 & -0.013525856 \\
\hline 379 & -0.215193187 & 0.175042714 \\
\hline 380 & 0.121433585 & 0.077487609 \\
\hline 381 & 0.284187118 & 0.083225072 \\
\hline 382 & 0.112457676 & -0.106054998 \\
\hline 383 & -0.037013723 & -0.10612353 \\
\hline 384 & 0.050241698 & -0.158806749 \\
\hline 385 & -0.121434625 & 0.077478054 \\
\hline 386 & 0.220117025 & 0.299738114 \\
\hline 387 & 0.110266182 & 0.070313337 \\
\hline 388 & 0.092290004 & -0.105932539 \\
\hline 389 & -0.102667012 & 0.115059049 \\
\hline 390 & 0.037503353 & -0.140125007 \\
\hline 391 & -0.065441364 & -0.160088725 \\
\hline 392 & -0.036504701 & 0.090984666 \\
\hline 393 & -0.066814631 & -0.082792078 \\
\hline 394 & -0.120233018 & 0.055599123 \\
\hline 395 & -0.106520078 & 0.183584566 \\
\hline 396 & -0.024603454 & 0.051494992 \\
\hline 397 & -0.19913509 & 0.140531387 \\
\hline 398 & 0.07122088 & -0.202081758 \\
\hline 399 & -0.013807916 & 0.0809354 \\
\hline 400 & 0.081953205 & 0.242763614 \\
\hline
\end{tabular}

